\title{
An all-digital PLL with supply insensitive digitally controlled oscillator
}

\author{
Seong-Young Seo ${ }^{1,2 a)}$, Jung-Hoon Chun ${ }^{1}$, Young-Hyun Jun ${ }^{2}$, \\ and Kee-Won Kwon ${ }^{1 \mathrm{~b})}$ \\ ${ }^{1}$ College of Information and Communication Engineering, Sungkyunkwan \\ Univesity, 2066, Seobu-ro, Jangan-gu, Suwon, Gyeonggi-do 440-746, Korea \\ ${ }^{2}$ Memory Division, Samsung Electronics Co., \\ 1, Samsungjeonja-ro, Hwaseong, Gyeonggi-do 445-701, Korea \\ a)seongyoungseo0@gmail.com \\ b)keewkwon@skku.edu
}

Abstract: This paper presents a divider-less all-digital PLL (ADPLL) with supply insensitivity. We employ a feed-forward inverter to make the oscillator insensitive to supply variation and utilize the delta-sigma modulation to improve the resolution. The onchip calibration tracks the optimum compensation strength for process and nominal voltage variations. We use an asynchronous counter to decide the phase error for low power. The proposed ADPLL was fabricated in a $0.13 \mu \mathrm{m}$ CMOS process. The silicon area of the ADPLL is $0.26 \mathrm{~mm}^{2}$ and the power consumption is $5.8 \mathrm{~mW}$ at $320 \mathrm{MHz}$. The spur level with the proposed compensation scheme was improved from $-57 \mathrm{dBc}$ to $-84 \mathrm{dBc}$ with an intentional supply noise. Keywords: ADPLL, DCO, supply sensitivity, feed-forward inverter Classification: Integrated circuits

\section{References}

[1] J. Li, N. Ning, L. Du, Q. Yu, and Y. Liu, “The Impact of Gate Leakage Current on PLL in $65 \mathrm{~nm}$ Technology: Analysis and Optimization," $J$. Semiconductor Technology and Science, vol. 12, no. 1, pp. 99-106, March 2012.

[2] R. B. Staszewski and P. T. Balsara, "Phase-Domain All-Digital PhaseLocked Loop," IEEE Trans. Circuits Syst. II, Exp. Briefs, vol. 52, no. 3, pp. 159-163, March 2005.

[3] L. Xu, S. Lindfors, K. Stadius, and J. Ryynänen, "A 2.4-GHz Low-Power All-Digital Phase-Locked Loop,” IEEE J. Solid-State Circuits, vol. 45, no. 8, pp. 1513-1521, Aug. 2010.

[4] E. Temporiti, C. Weltin-Wu, and F. Svelto, "A 3 GHz Fractional AllDigital PLL with a $1.8 \mathrm{MHz}$ Bandwidth Implementing Spur Reduction Techniques," IEEE J. Solid-State Circuits, vol. 44, no. 3, pp. 824-834, July 2009.

[5] V. Kratyuk, P. K. Hanumolu, U.-K. Moon, and K. Mayaram, “A Design Procedure for All-Digital Phase-Locked Loops Based on a Charge-Pump Phase-Locked-Loop Analogy," IEEE Trans. Circuits Syst. II, Exp. Briefs, vol. 54, no. 3, pp. 247-251, March 2007.

[6] S.-Y. Seo, J.-H. Chun, Y.-H. Jun, S. Kim, and K.-W. Kwon, "A Digitally 
Controlled Oscillator with Wide Frequency Range and Low Supply Sensitivity," IEEE Trans. Circuits Syst. II, Exp. Briefs, vol. 58, no. 10, pp. 632-636, Oct. 2011.

[7] R. B. Staszewski, C. Hung, N. Barton, M. Lee, and F. Svelto, “A Digitally Controlled Oscillator in a $90 \mathrm{~nm}$ Digital CMOS Process for Mobile Phones," IEEE J. Solid-State Circuits, vol. 40, no. 11, pp. 2203-2211, Nov. 2005.

[8] R. B. Stasewski, S. Vemulapalli, P. Vallur, J. Wallberg, and P. T. Balsara, "1.3 V 20 ps Time-to-Digital Converter for Frequency Synthesis in 90-nm CMOS," IEEE Trans. Circuits Syst. II, Exp. Briefs, vol. 53, no. 3, pp. 220224, March 2006.

[9] I.-C. Hwang, C.-W. Kim, and S.-M. Kang, “A CMOS Self-Regulating VCO with Low Supply Sensitivity," IEEE J. Solid-State Circuits, vol. 39, no. 1, pp. 42-48, Jan. 2004.

[10] B.-M. Moon, Y.-J. Park, and D.-K. Jeong, "Monotonic Wide-Range Digitally Controlled Oscillator Compensated for Supply Voltage Variation,” IEEE Trans. Circuits Syst. II, Exp. Briefs, vol. 55, no. 10, pp. 1036-1040, Oct. 2008.

\section{Introduction}

In a deep sub-micron process technology, it is difficult to design a conventional charge-pump-based PLL with analog loop filter (LF), due to small voltage headroom and poor signal-to-noise ratio (SNR). In particular, the gate leakage of the loop filter causes ripples of the voltage controlled oscillator (VCO) input [1], and consequently generates reference spurious tones in the frequency spectrum of the output. It also takes a long time to design a new circuit or to revise the existing version for the next generation process with the analog components. Various all-digital PLLs, which make the best use of digital signal processing around the loop, have been developed recently $[2,3,4,5]$ to overcome aforementioned problems.

Digital circuits, however, deteriorate the phase noise performance because of its finite resolution. The quantization error of the time-to-digital converter (TDC) worsens in-band phase noise [4]. And the discrete characteristics of a digitally controlled oscillator results in out-of-band phase noise.

We adopted divider-less architecture among digital PLLs which has relatively fast settling time. Fig. 1 shows a typical divider-less ADPLL. It has a linear phase detector (LPD), which can calculate the phase difference between the reference and oscillator clocks in a unit of the oscillator clock

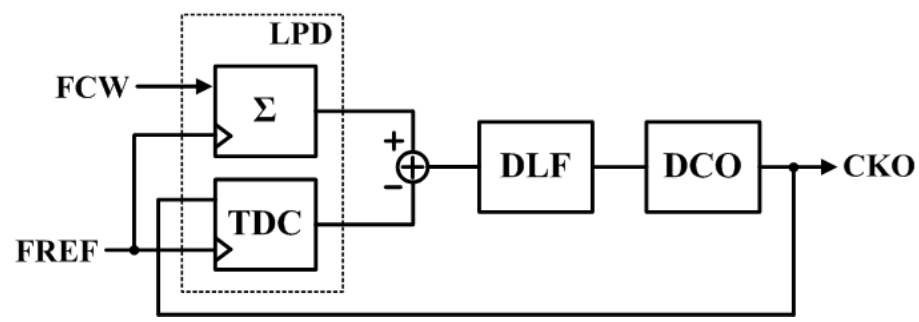

Fig. 1. Typical divider-less All-Digital PLL. 
period, independent of the amounts of the phase error $[2,3,4]$. We propose a simple phase detector (PD) operating in the manner of interleaving of two asynchronous down counters, which consumes smaller area and dissipates less power compared to synchronous accumulators.

Most of efforts are focused on improving the DCO. We chose the ringtopology as the DCO architecture because an LC oscillator is too bulky. A hierarchical structure, consisting of coarse and fine delay stages, is used to achieve wide frequency range as well as fine resolution. The supply compensation scheme is also adopted to alleviate the inherent supply sensitivity of the ring-type oscillator. We realized the supply insensitive DCO by utilizing a feed-forward inverter to compensate for supply variation [6] and utilized the delta-sigma modulator (DSM) to improve the resolution further. The on-chip calibration logic adaptively tracks the optimum compensation strength for process and supply variations.

\section{Digitally controlled oscillator}

\subsection{Supply compensation and on-chip calibration}

The proposed DCO architecture is shown in Fig. 2. Because DCOs in ring topology are very susceptible to supply noise, we used a supply compensation scheme using a feed-forward inverter [6]. The feed-forward compensator, marked as "C", transports the signals before the two consecutive main inverters, marked as "M", do with opposite polarity. If the delay variation of the compensator is comparable to that of the main inverter, the supply dependency is removed, so that the tap-to-tap delay remains unchanged. The tap-to-tap delay is small since signals in this chain do not swing full scale of the supply. The single-ended inverter chain occupies only about half of the silicon area and consequently dissipates less power when compared to the conventional pseudo differential chain with a latch for supply compensation [10].

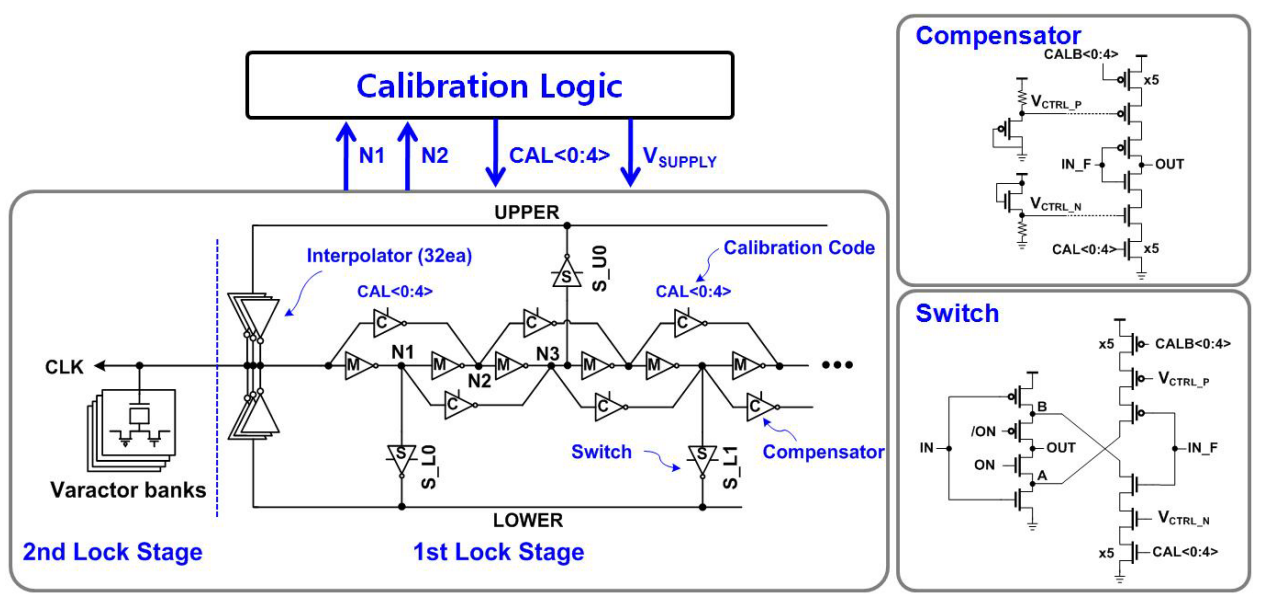

Fig. 2. The architecture of the digitally controlled oscillator, supply compensator and the structure of the switch.

As shown in Fig. 2, we chose a current starved inverter as the compensator since its delay variation is compensated with a small size of compensator. And the bias voltages $\mathrm{V}_{\mathrm{CTRL} \_\mathrm{P}}$ and $\mathrm{V}_{\mathrm{CTRL} \_\mathrm{N}}$ are generated by 
simple diode-connected transistors to lower the gate overdrive of the compensator and generate proportional voltage to the supply instead of using analog circuits. These control voltages are connected commonly to all the compensators. Since the switch is susceptible to supply fluctuation as well, the similar compensation is included. Two series of pMOS and nMOS transistors are connected to the node $\mathrm{A}$ and $\mathrm{B}$, respectively. The input marked as IN_F is fed from the previous node of the delay chain. For example, when the switch, S_U0, is selected, IN and IN_F are connected to N3 and N2 nodes, respectively. In this way, the capacitive loading of UPPER and LOWER node is minimized because the compensators of unselected switches have no influence on UPPER and LOWER nodes. Both the strength of the compensators and switches are controlled by the 5-bit binary code depicted by $\mathrm{CAL}<0: 4>$. It is difficult to optimize the strength of them across the process, voltage and temperature variations. Therefore, the on-chip calibration logic is prepared.

Fig. 3(a) shows the calibration logic for the supply compensation. In general, the frequency of a ring oscillator increases with the supply voltage when compensation is negligible. As the compensation gets stronger by sweeping CAL, the dependency of frequency on the supply voltage decreases, and $d f / d V$ turns to negative at the critical strength of compensation $(\mathrm{CAL}<\mathrm{cr}>)$. At $\mathrm{CAL}<\mathrm{cr}>$ or $\mathrm{CAL}<\mathrm{cr}-1>$, the impact of the supply variation, $|d f / d V|$, is the minimum. In this work, there are two levels of power supply of the DCO, $\mathrm{V}_{\mathrm{DD}}$ and $\mathrm{V}_{\mathrm{DDL}}\left(10 \%\right.$ lower than $\mathrm{V}_{\mathrm{DD}}$ ) which are alternated by $\mathrm{V}_{\mathrm{CON}}$. The TDC measures delay between the rising edges of N1 and N2. Because N1 and N2 are complementary as shown in Fig. 2, the delay is estimated to $\left(T / 2+t_{d}\right)$ where $T$ is the period of the

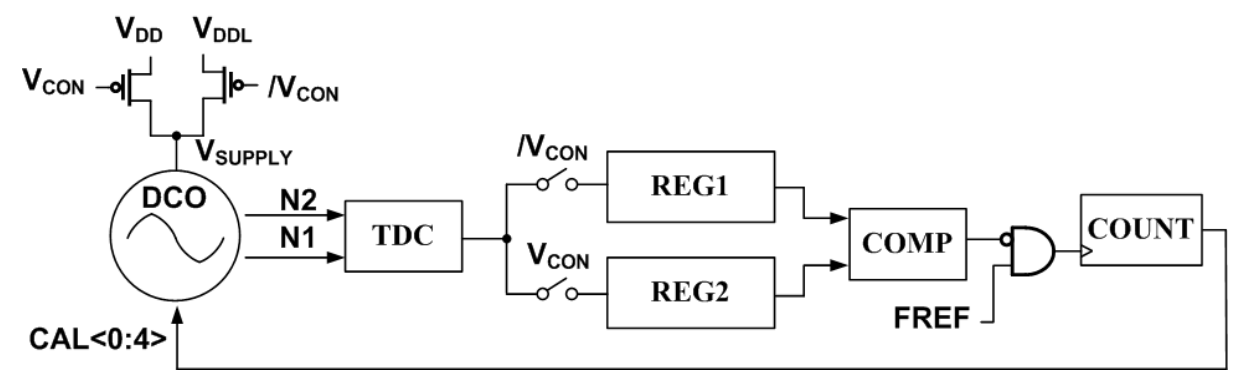

(a)

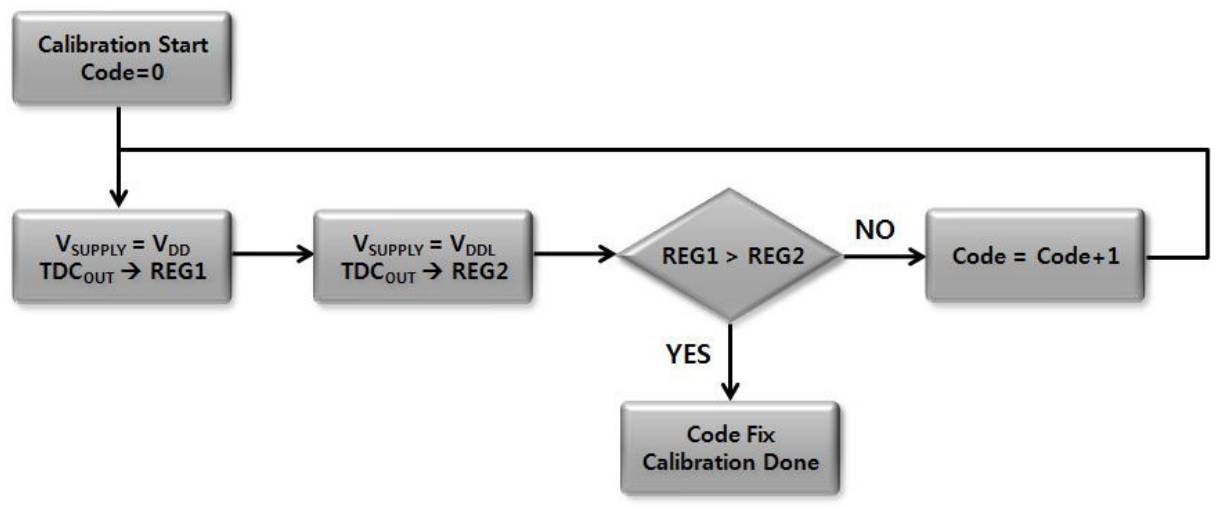

(b)

Fig. 3. (a) The on-chip calibration logic for the supply compensation and (b) the flow chart of the calibration process. 
oscillator and $t_{d}$ is the delay of a main inverter. The TDC outputs are stored to REG1 and REG2 when $\mathrm{V}_{\text {SUPPLY }}$ is $\mathrm{V}_{\mathrm{DD}}$ and $\mathrm{V}_{\mathrm{DDL}}$, respectively. Fig. $3(\mathrm{~b})$ shows the flow chart of the calibration process. As long as COMP decides that REG1 is not bigger than REG2, COUNT increases the binary code of the compensator, CAL. At CAL $<$ cr $>$, REG1 surpasses REG2. Since the binary code of this moment is close to the optimum strength of the compensator, the calibration process is terminated and $\mathrm{CAL}<\mathrm{cr}>$ is stored. The resolution of the TDC is desired to be finer than the delay variation between the two rising edges of N1 and N2 by a unit step of CAL in order to avoid the same codes in REG1 and REG2 over several CALs. The delay of rising edges from $\mathrm{N} 1$ to $\mathrm{N} 2$ represents the whole ring delay including any possible mismatch between stages. If the calibration is repeated with the falling edges at the same nodes, the representativeness may be improved. The calibration operation can be executed during the power-up sequence or idle status. The temperature variation due to operating conditions can be further compensated by quick update of CAL during normal operation with minimum timing overhead.

In Fig. 4(a), the simulation result of the calibration process is shown. When $\mathrm{V}_{\text {SUPPLY }}$ toggles between $\mathrm{V}_{\mathrm{DD}}$ and $\mathrm{V}_{\mathrm{DDL}}$, the frequency of the clock is proportional to the power supply. At the critical point, the frequency increases with decrease of power supply. Fig. 4(b) shows the normalized frequency variations along with the normalized perturb of the supply voltage. As shown in the figure, the normalized frequency variation, in the

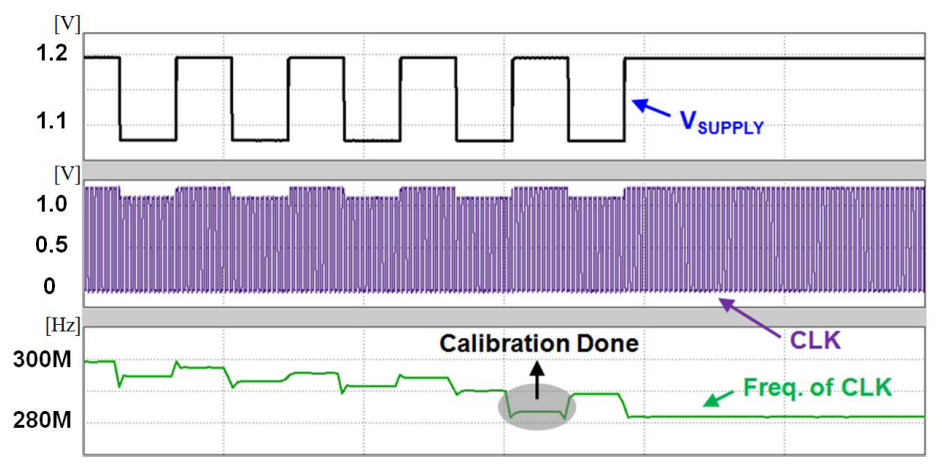

(a)

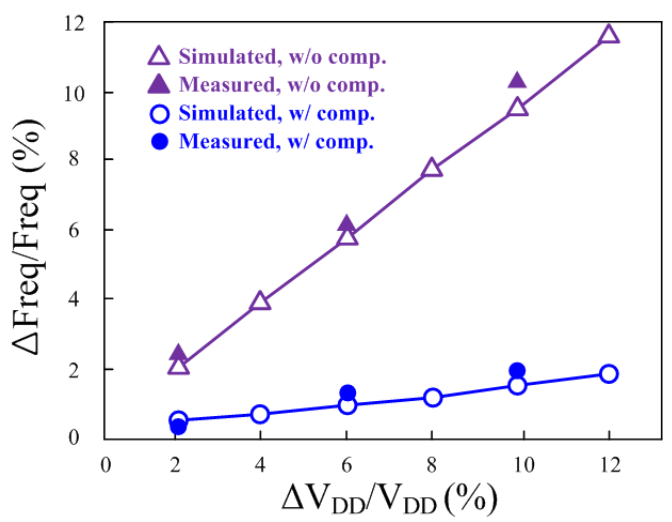

(b)

Fig. 4. (a) Simulation results of the calibration process and (b) the simulated and measured frequency variation due to the supply fluctuation. 
case of not using the compensation, is around $2 \%$ and $10 \%$, when $\Delta \mathrm{V}_{\mathrm{DD}} /$ $\mathrm{V}_{\mathrm{DD}}$ is $2 \%$ and $10 \%$, respectively. The supply insensitivity is significantly improved in the case of utilizing the proposed compensation scheme and the normalized frequency changes are around $0.4 \%$ and $1.5 \%$ at the same $\mathrm{V}_{\mathrm{DD}}$ variations. The supply sensitivity is estimated as low as $0.15 \%-\mathrm{f}_{\mathrm{DCO}} /$ $1 \%$-supply on average in this work. The measurements of the normalized frequency variation in the free-running status depending on the various supply voltages are also included as filled symbols in Fig. 4(b). The measured immunity to supply change agrees with the simulated estimation.

Fig. 5 shows the simulation results of the DCO supply sensitivity to the process corners and temperatures with the calibration logic. As shown in Fig. 5(a), the sensitivity in fast, typical and slow corners is $0.13,0.15$ and $0.17 \%-\mathrm{f}_{\mathrm{DCO}} / 1 \%-\mathrm{V}_{\mathrm{DD}}$, respectively. If we fix the binary code of the compensator with the value obtained from the calibration at typical process, the supply sensitivity of the DCO increases up to 0.23 and $-0.33 \%$ $\mathrm{f}_{\mathrm{DCO}} / 1 \%-\mathrm{V}_{\mathrm{DD}}$, at fast and slow corners, respectively. Fig. $5(\mathrm{~b})$ shows supply sensitivity at different temperatures. Although the critical code at room temperature is used at hot or cold temperature, the sensitivity is deteriorated to $0.3 \%-\mathrm{f}_{\mathrm{DCO}} / 1 \%-\mathrm{V}_{\mathrm{DD}}$ or less. Because the difference is small, the sensitivity can be reduced again by quick update during the normal operation of ADPLL.

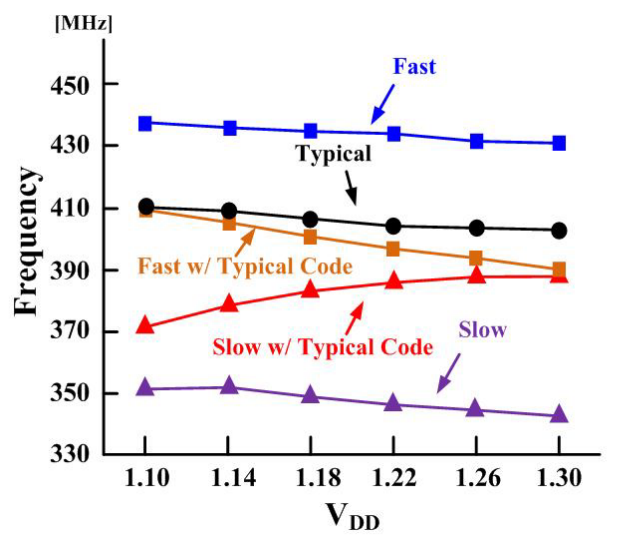

(a)

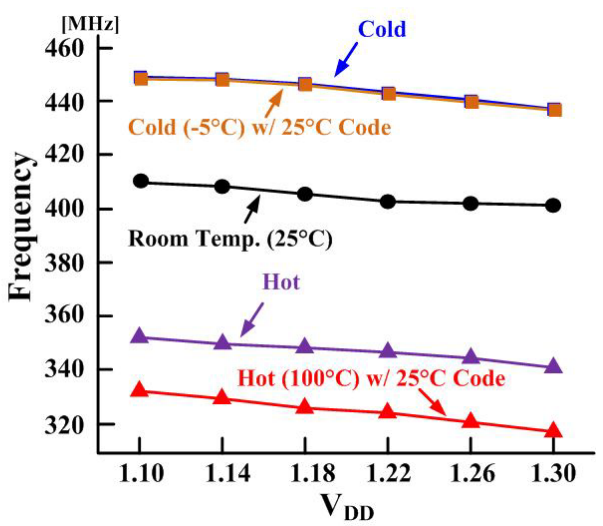

(b)

Fig. 5. The simulated DCO frequency due to supply variation at various (a) process corners and (b) temperatures.

\subsection{Wide frequency range and fine resolution}

The entire tuning structure is divided into a coarse locking stage, and a fine locking stage. The coarse locking stage consists of the main inverter delay chain, the switches, and the interpolators. The switches are used to select tapping nodes, where "UPPER" and "LOWER" are connected. This hierarchical structure allows us to achieve a wide frequency range from $170 \mathrm{MHz}$ to $1.1 \mathrm{GHz}$ with eight coarse stages. The interpolators are implemented to increase the frequency resolution of the first locking stage, which is one sixteenth of a tap-to-tap delay. The fine stage of locking is accomplished by two varactor banks. An nMOS is used as a varactor, and the deep n-well is used to block the substrate noise from the other digital blocks. One varactor bank is needed to track the integer part of the phase 
error. There are 31 varactors in this bank, and 32 states controlled by pseudo thermometer code. The period tuning range of the second locking stage is $250 \mathrm{ps}(+/-125 \mathrm{ps})$. This tuning range is wide enough to cover the resolution of the first stage of locking, which is $13 \mathrm{ps}$. Therefore, the loop can lock, even if the switchover from the first phase into the second phase is done with the residue of the phase error. The other varactor bank was implemented to apply the delta-sigma modulation (DSM). The varactor in this bank is exactly the same as that of the bank of the integer part, and is controlled in the fashion of the summation of each capacitance of the varactors. So, it is possible that the outputs of the DSM are directly fed to the DCO. This method has the advantage of not having a summing circuit to consume large area and power dissipation. Since it does not change other integer parts of the DCO control bits, the digital switching noise is also reduced [7].

\section{Proposed divider-less ADPLL}

Fig. 6 shows the proposed divider-less ADPLL. It consists of a phase detector (PD), a digital loop filter (DLF) and a DCO. The PD detects not only the integer part of the phase error, by counting the number of positive edges of the oscillator clocks (CKO) during a period of the reference clock (FREF), but also the fractional part of it, using a TDC, which figures out the position of the positive edge of the FREF, within a period of the CKO. We employ a simple asynchronous down counter operating in an interleaving way, for manipulating the integer part of the phase error, instead of using bulky accumulators, resulting in low power consumption and compact design. Fig. 7(a) shows the waveforms of critical signals of the interleaving scheme. Assuming that FCW is four, for instance, the frequency of CKO is four times as high as that of FREF. This kind of phase detector is not completely linear, but is linear if $2 \cdot \mathrm{T}_{\mathrm{CKO}} \cdot \mathrm{FCW}$ is smaller than $\mathrm{T}_{\mathrm{FREF}}$, where $\mathrm{T}_{\mathrm{CKO}}$ is the averaged period of the oscillator clock, and $\mathrm{T}_{\mathrm{FREF}}$ is the period of the reference clock. The block named Retimed Clock carries out synchronizing FREF to CKO, and transmitting pulses into the down counters, to calculate the integer part of the phase error. During one counter running, the other is forced to be in an initial condition by the reset signal. The block of phase detecting can operate properly at a relaxed speed in this fashion. An asynchronous counter which

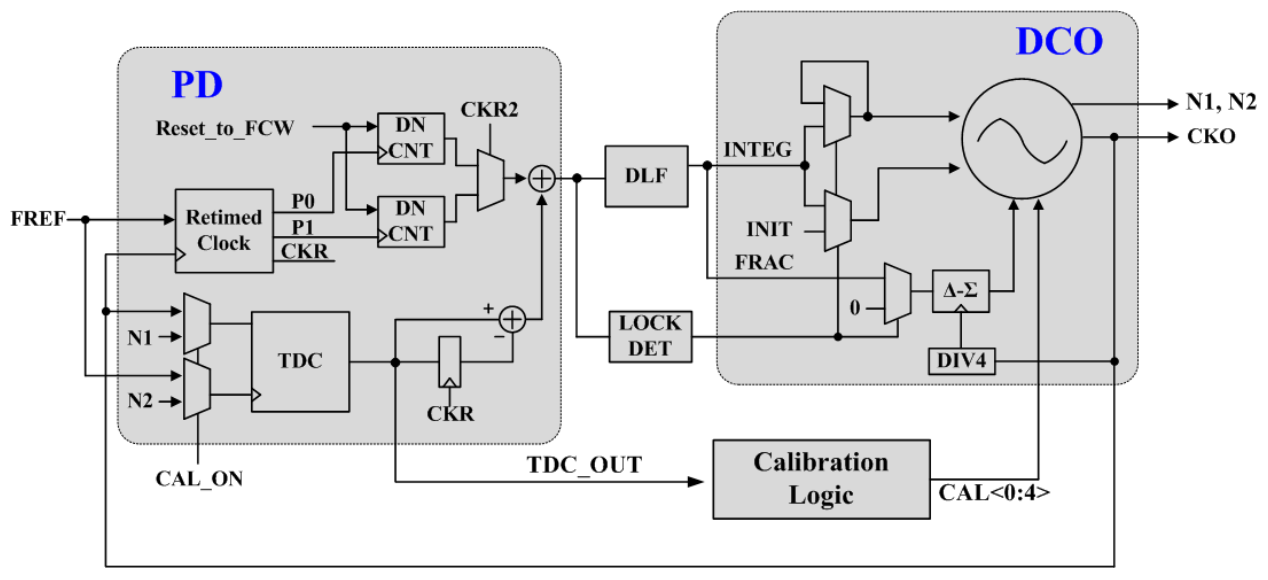

Fig. 6. The architecture of the proposed ADPLL. 


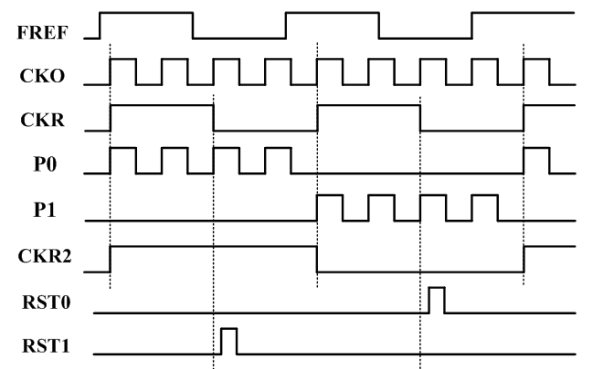

(a)

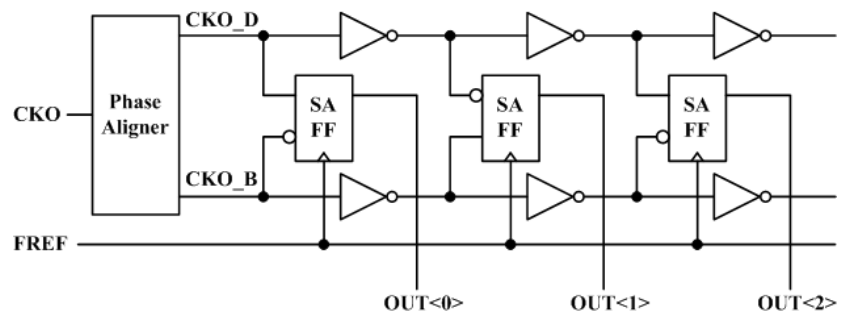

(b)

Fig. 7. (a) The waveforms of the critical signals in the proposed linear phase detector and (b) the timeto-digital converter in this work.

occupies small silicon area and consumes relatively less power is therefore enough for this work. In Fig. 7(b), the TDC is implemented to improve the resolution of the $\mathrm{PD}$, and to grab the position of the reference clock within the oscillator clock. A sense-amplifier type flip-flop was used as a capture unit to reduce the uncertainty window [8]. The resolution of the TDC is one inverter delay which is $58 \mathrm{ps}$ in this work. Since the number of stages is 128 , the TDC can operate up to around $7.4 \mathrm{~ns}$. The TDC is also used for the calibration process and decides the time difference between two representative signals from the DCO. The calibration logic uses the output of the TDC and tunes the binary code of the compensator in the DCO. The locking process is composed of two stages - a coarse and fine stage to achieve both the wide frequency range, and the fine resolution. A lock detector is used to switch the locking stage.

\section{Measurement}

We fabricated the proposed ADPLL using $0.13 \mu \mathrm{m}$ CMOS technology. A die micrograph is shown in Fig. 8. To create a supply noise environment, we removed the supply decoupling capacitors around the core of the DCO.

Fig. 9 shows the comparison of the DCO phase noise characteristics between the cases with, and without, compensations. We applied the $100 \mathrm{mVp}-\mathrm{p}, 1 \mathrm{MHz}$ sinusoidal noise at the supply using a coupling capacitor to put a noise tone out of the bandwidth. When using the supply compensation scheme, the out-of-band phase noise performance was improved significantly. The phase noise level with the compensation scheme was improved from $-57 \mathrm{dBc}$ to $-84 \mathrm{dBc}$. The spurious tones due to the harmonics of the supply noise were also reduced. The phase noise is $-101.4 \mathrm{dBc} / \mathrm{Hz}$ at $1-\mathrm{MHz}$ frequency offset from the carrier frequency of $320 \mathrm{MHz}$. Fig. 10 shows the frequency spectrum, depending on the use of the 


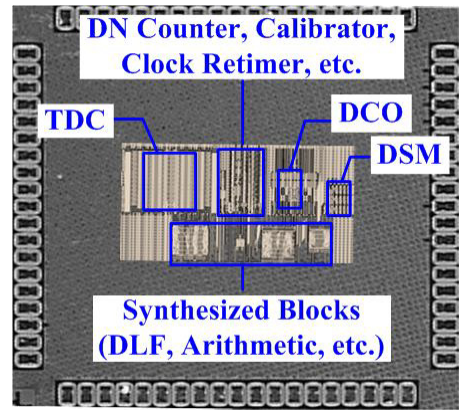

Fig. 8. Chip micrograph of the proposed ADPLL fabricated using $0.13 \mu \mathrm{m}$ CMOS technology.

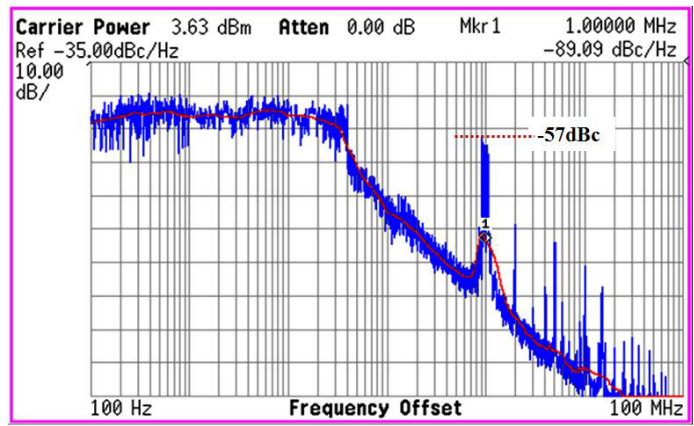

(a)

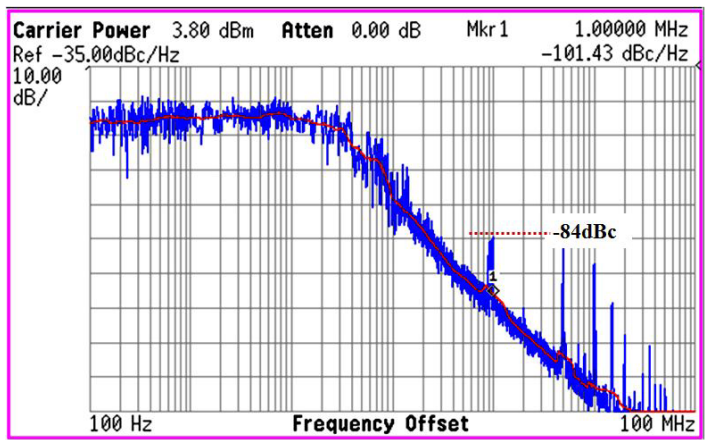

(b)

Fig. 9. SSB phase noise of the DCO (a) without, and (b) with the supply compensation at the carrier frequency of $320 \mathrm{MHz}$.

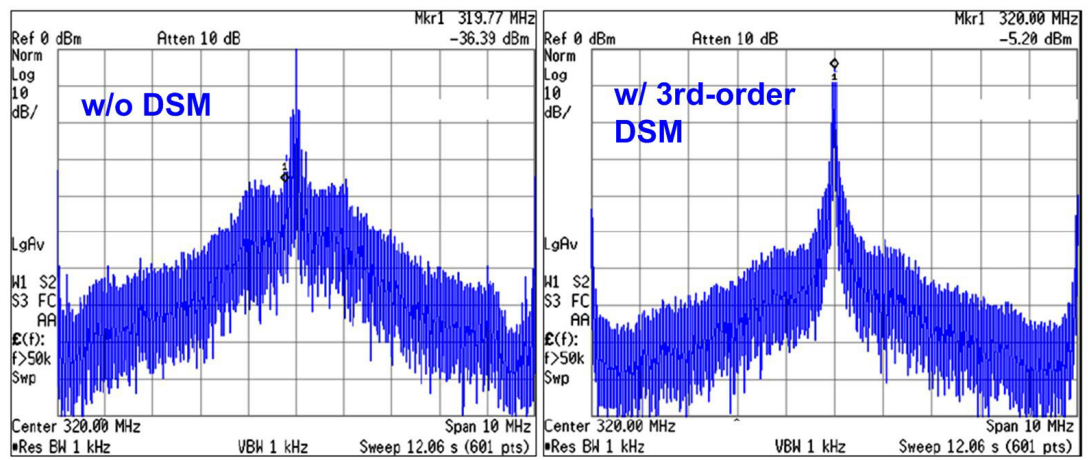

Fig. 10. Frequency spectra at the carrier frequency of $320 \mathrm{MHz}$ without the DSM and with the thirdorder DSM. 
DSM. The reference and target frequencies are $5 \mathrm{MHz}$ and $320 \mathrm{MHz}$, respectively. The dithering frequency is one fourth of carrier frequency. As shown, the frequency spectrum without the DSM is spread out severely in the vicinity of the carrier frequency. On the other hand, the case of using the third-order DSM improves the degree of spreading distinctly, and the levels of the frequency components near the carrier frequency. The phase noise of higher frequencies over $640 \mathrm{MHz}$ was not measured properly because there is a signal race problem at the phase detector.

The silicon area that the entire chip occupies is $0.26 \mathrm{~mm}^{2}$ while the DCO core takes up $0.006 \mathrm{~mm}^{2}$. The DCO of the ADPLL works in the frequency range from $170 \mathrm{MHz}$ to $1.1 \mathrm{GHz}$. The settling time was $6 \mu \mathrm{s}$ (60 cycles) with $10 \mathrm{MHz}$ of the reference frequency. The power consumption is $5.8 \mathrm{~mW}$ at the DCO frequency of $320 \mathrm{MHz}$. The DCO consumes only $2.88 \mathrm{~mW}$ because a single-ended topology is used instead of the pseudo differential structure in the coarse tuning stage. The performance summary of the proposed ADPLL and the related works is shown in Table I. The supply sensitivity was improved when compared to [10]. Although [9] that is a VCO implementation seems good, the sensitivity varies with the control voltage of VCO. Since the number of the stages is reduced at high frequencies, the power consumption of the proposed DCO inversely dependent on the frequency.

Table I. Summary of the proposed ADPLL performance.

\begin{tabular}{|c|c|c|c|}
\hline & [9] & [10] & This work \\
\hline Process & $0.35 \mu \mathrm{m}$ CMOS & $0.13 \mu \mathrm{m}$ CMOS & $0.13 \mu \mathrm{m}$ CMOS \\
\hline Supply & $3.3 \mathrm{~V}$ & $1.28 \mathrm{~V}$ & $1.2 \mathrm{~V}$ \\
\hline $\begin{array}{l}\text { Acquisition } \\
\text { Time }\end{array}$ & N/A & Open Loop & $6 \mu \mathrm{s}(60 \mathrm{cycles})$ \\
\hline Tuning Range & $4 \mathrm{KHz} \sim 1.1 \mathrm{GHz}$ & $300 \mathrm{MHz} \sim 1.3 \mathrm{GHz}$ & $170 \mathrm{MHz} \sim 1.1 \mathrm{GHz}$ \\
\hline $\begin{array}{c}\text { Supply } \\
\text { Sensitivity of } \\
\text { DCO }\end{array}$ & $0.15 \%$-delay $/ 1 \%$ - $\mathrm{V}_{\mathrm{DD}}$ & $0.2 \%$-period $/ 1 \%-\mathrm{V}_{\mathrm{DD}}$ & $0.15 \%-\mathrm{f}_{\mathrm{DCO}} / 1 \%-\mathrm{V}_{\mathrm{DD}}$ \\
\hline $\begin{array}{c}\text { Power } \\
\text { Consumption }\end{array}$ & $\begin{array}{c}28 \mathrm{~mW} \\
@ 900 \mathrm{MHz}\end{array}$ & $\begin{array}{c}\text { DCO } 4.48 \mathrm{~mW} \\
@ 950 \mathrm{MHz}\end{array}$ & $\begin{array}{c}5.8 \mathrm{~mW} \\
\text { (DCO } 2.28 \mathrm{~mW} \text {, } \\
\text { Counter } 1.8 \mathrm{~mW} \text { ) } \\
\text { @ } 320 \mathrm{MHz}\end{array}$ \\
\hline Silicon Area & $0.12 \mathrm{~mm}^{2}$ & $\begin{array}{c}0.007 \mathrm{~mm}^{2} \\
\text { (DCO core) }\end{array}$ & $0.26 \mathrm{~mm}^{2}$ \\
\hline $\begin{array}{l}\text { Phase Noise } \\
\text { or rms Jitter }\end{array}$ & $\begin{array}{c}-101.4 \mathrm{dBc} / \mathrm{Hz} \\
\text { @600kHz offset from } \\
900 \mathrm{MHz}\end{array}$ & $\begin{array}{c}10.4 \mathrm{ps} \\
@ 950 \mathrm{MHz}\end{array}$ & $\begin{array}{c}-101.4 \mathrm{dBc} / \mathrm{Hz} \\
\text { @ } 1 \mathrm{MHz} \text { offset from } \\
320 \mathrm{MHz}\end{array}$ \\
\hline
\end{tabular}

\section{Conclusion}

We have presented an ADPLL that includes a supply compensation and delta-sigma modulation to improve the phase noise performance of the DCO. The on-chip calibration for supply compensation is employed to search the optimum compensation conditions for the process and the nominal voltage variations. Two asynchronous down counters operating in an interleaving way were used to calculate the integer part of the phase error for low power. We fabricated the proposed ADPLL using 0.13- $\mu \mathrm{m}$ CMOS technology. The silicon area that the ADPLL occupies is $0.26 \mathrm{~mm}^{2}$, 
and the power consumption at $320 \mathrm{MHz}$ is $5.8 \mathrm{~mW}$. The frequency tuning range covers from $170 \mathrm{MHz}$ to $1.1 \mathrm{GHz}$. The phase spur level with the compensation scheme was improved from $-57 \mathrm{dBc}$ to $-84 \mathrm{dBc}$. The phase noise is $-101.4 \mathrm{dBc} / \mathrm{Hz}$, at $1-\mathrm{MHz}$ frequency offset from the carrier frequency of $320 \mathrm{MHz}$.

\section{Acknowledgements}

This work was supported by the Information Technology Research and Development Program of the Ministry of Knowledge Economy of Korea/ Institute of Information Technology Advancement under Grant 2009-F015-01, and in part by the IC Design Education Center at the Korea Advanced Institute of Science and Technology. 